\title{
Fabrication, Visualization and Analysis of Fluorescein Sodium Encapsulated PLGA@CS Nanoparticles as Model for Photothermomechanical Drug Delivery Using Pulsed 532 nm Laser
}

\author{
Mohammad E. Khosroshahi1,2,3*, Mahboobeh Mahmoodi, \\ ${ }^{1}$ Laser \& Nanobiophotonics Laboratory, Faculty of Biomedical Engineering, Amirkabir University of Technology, Tehran, Iran \\ ${ }^{2}$ MIS-Electronics, Nanobiophotonics \& Biomedical Research Laboratory, Richmond Hill, Canada \\ ${ }^{3}$ Department of Mechanical and Industrial Engineering, University of Toronto, Toronto, Canada \\ ${ }^{4}$ Department of Biomedical Engineering, Azad University, Yazd Branch, Yazd, Iran \\ Email: *khosrom@mie.utoronto.ca
}

How to cite this paper: Khosroshahi, M.E. and Mahmoodi, M. (2018) Fabrication, Visualization and Analysis of Fluorescein Sodium Encapsulated PLGA@CS Nanoparticles as Model for Photothermomechanical Drug Delivery Using Pulsed $532 \mathrm{~nm}$ Laser. Advances in Nanoparticles, 7, 47-68. https://doi.org/10.4236/anp.2018.73005

Received: July 6, 2018

Accepted: August 28, 2018

Published: August 31, 2018

Copyright $\odot 2018$ by authors and Scientific Research Publishing Inc. This work is licensed under the Creative Commons Attribution International License (CC BY 4.0).

http://creativecommons.org/licenses/by/4.0/

c) (i) Open Access

\begin{abstract}
PLGA/CS nanoparticles containing fluorescein sodium as drug model were synthesized and characterized to investigate the feasibility of laser-induced drug delivery using pulse $532 \mathrm{~nm}$. The main objective was to investigate the photothermally-induced mechanical force for transporting the nanoparticles. An argon laser was used to excite the fluorescence of the samples after irradiation. The preliminary results indicated that the drug nanoparticles encapsulated trapped by the cavitation bubbles can be transported by photothermomechanical effect. Different regions of interactions are defined and while in our case, the thermoelastic does not apply due to higher fluences, vaporization and laser-induced thermal breakdown (LITB) including the plasma formation and shock waves played an important and major role. Threshold fluences of 2.8, 18 and $102 \mathrm{Jcm}^{-2}$ corresponding to $0.28,1.8$ and $10 \mathrm{GWcm}^{-2}$ and 3.8, 30, and $171 \mathrm{MPa}$ are determined for ablation, vaporization and LITB mechanisms respectively. The secondary microbubbles due to explosion of the primary transient cavitation bubbles played a key role in delivery process. Despite the dominant argon laser brightness, the laser-induced fluorescence spectroscopy (LIFS) demonstrated the fluorescence emission of the cavitation bubbles carrying due to the drug nanoparticles entrapped within the biogelatin after exposure to laser radiation, the irradiation, which confirms the possibility of transport of drug nanoparticles by laser cavitation. Finally, it is
\end{abstract}


suggested that the nature of such photothermal and photo non-thermal mechanical effects is governed and influenced by determining and criticizing in terms of the type of nanomaterial as well as their synthesis process engineering and fabrication as they can be made case sensitive by selecting different types of materials for a specific application.

\section{Keywords}

Drug Delivery, Nanoparticles, Laser, Fluorescence, Photothermomechanical Effect, Cavitation Bubbles

\section{Introduction}

It is well established that the drug delivery systems (DDS) can enhance the efficacy of different pharmaceutical payloads while improving the poor solubility, limited stability, biodistributions as well as pharmacokinetics of drug [1] [2]. However, one major issue in any DDS is the fate of the drug inside the body where it is aimed to minimize the side effects and enhance the effectiveness of drug delivery. To achieve these goals, an understanding of physiology, biophysics and the drug movement within tissue is essential. Traditional DDS include oral and intravenous (IV) routes of administration where the former via tablets or capsules is largely inefficient due to exposure of drug to the metabolic processes of the body [3] and thus a greater amount of drug is required to meet the effectiveness, which in turn has an uptake limitation, and the latter can also be an issue because high amount of required drug create high concentration of the drug in the blood stream hence producing a toxic side effects [4]. Engineered nanomaterials hold great promise in DDS [5] [6] such as liposomes, metallic/nonmetallic, and polymeric nanoparticles have increased the ability to deliver drugs for which conventional therapies have shown limited efficacy. These nanoparticles can either be made passive (i.e., based on the enhanced permeation and retention (EPR) effect) or active where they are functionalized with ligand to target specific tissues or cell types. Recently, we demonstrated that liposomes containing doxorubicin as cancer drug and magnetoplasmonics can be used as passive [7] and active [8] DDS for cancer hyperthermia.

The main advantages of polymeric nanoparticles (PNP) as DDS are that they increase the stability of any volatile pharmaceutical agents and are easily fabricated in considerable quantities. The PNP can be absorbable or degradable polymer, which allows the design of individual delivery system for highly specific applications. Among many, PLGA (Poly lactide-co-glycolide acid) which is hydrolytically unstable, insoluble in water and degraded by hydrolytic attack of ester bonds has been widely used in biomedical applications [9]. On the other hand, chitosan (CS) is an amino poly saccharide (Poly 1,4-D glucosamine), biocompatible, biodegradable and non-toxic polymer. Though, CS is a weak base and insoluble in water and organic solvents, it becomes soluble in dilute aqueous 
acidic solution with $\mathrm{pH}<6.5$. Additionally, CS enhances the drug transport across the cell membrane and has been extensively utilized in DDS and tissue engineering [10] [11]. The physical DDS are: 1) Electrical to drive molecules electrically through the skin (i.e., iontophoresis) or create pores within the membrane (i.e., electroporation) [12], 2) Ultrasound [13], Laser ultrasound [14], Extra corporeal shock waves [15], Laser-induced shock waves [16] and Laser-induced cavitation [17] [18] [19]. Recently, it was also shown that the mechanisms for the enhanced mobility and laser transport of NPs are thought to be due to electromagnetic wave induced force (i.e. an oscillatory motion) and laser absorptive force (i.e., photothermophoresis) where the laser beam trajectory path due to thermal heating results in the change of refractive index of medium and redistribution of NPs concentration [20].

Generally, the pulse laser interaction with absorbing liquid, gelatin or soft tissues takes place via different physical processes: 1) thermoelastic Figure 1(a) where the absorption of low energy short pulses i.e., shorter than acoustic transient times, followed by rapid expansion of heated volume generates a non-ablative bipolar thermoelastic stress waves (i.e., photoacoustic) within the medium. Acoustic transients are also produced by long pulses (i.e., $c_{a} \tau_{p} \gg \delta_{0}$ ) where $c_{\mathrm{a}}$ is acoustic velocity, $\tau_{p}$ is pulse duration and $\delta_{0}$ is the optical penetration within the medium respectively, due to the vapour plume and cavities. In the former case, the Gruneisen-stress produced by rapid heating cannot relax during the pulse hence a bipolar thermoelastic waves develops and propagates from the surface adjacent to air through the bulk of the medium [21] [22] [23]. As a result, the positive i.e., compression tensile stress travels through the medium and the negative tensile pulse reflects back toward the surface. If the tensile stress exceeds that of the material, it causes an internal rupture or spallation [24]. The importance of the thermoelastic stress generation is due to the fact that low hydrodynamic pressure below the equilibrium vapour pressure can create cavities filled with either vapour or gas. The increase of fluence in thermoelastic domain extends to the threshold of vaporization, 2) vaporization Figure 1(b) occurs at fluences higher than threshold fluence where the temperature is reached $100^{\circ} \mathrm{C}$ and superheating occurs. As a result, the target reactive forces due to escaping vapour exceed the thermoelastic limit hence giving rise to vapour cavity and the formation of microbubbles. The recoil momentum generated due to ablated material exerts stress on the material enhancing the built-up pressure within the bulk, and 3) Photomechanical (i.e. photodisruption) Figure 1(c) at higher values than vaporization where an optical dielectric breakdown occurs either due to multiphoton absorption or avalanche process, which in either case is accompanied by violent expulsion of liquid droplets, cavitation, the emission of sound, light due to plasma formation followed by generation of shock waves all at different time scale. Since, in our case the energy levels were above the ablation threshold thus 2) and 3) are the main mechanisms which sought to be responsible for the delivery of drug model [25] [26]. All above mechanisms have 

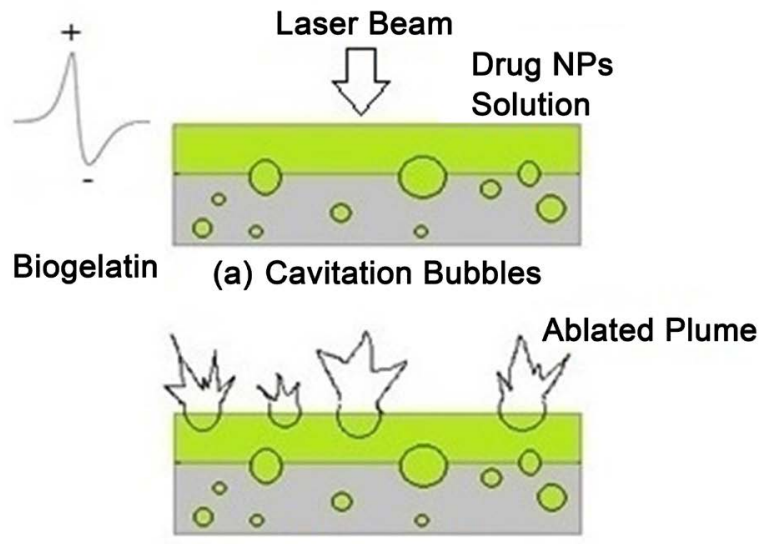

(b) Vaporization

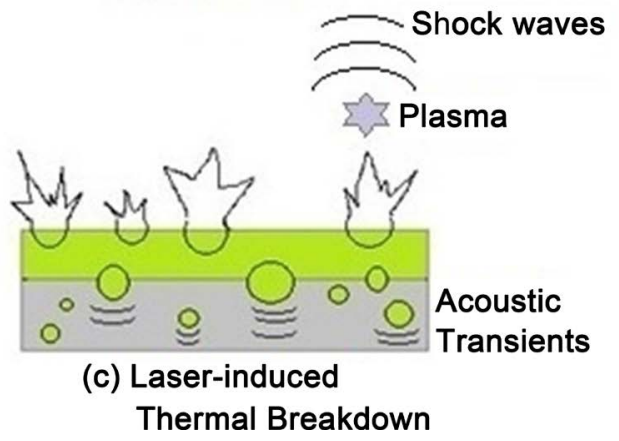

Figure 1. Schematic diagram of laser-biogel interaction process. (a) Cavitation bubbles; (b) Vaporization; and (c) Laser-induced thermal breakdown.

the potential to be utilized appropriately for a given biomedical application. For example, the outer layer of skin, the stratum corneum (SC) with a thickness of $10-20 \mu \mathrm{m}$ can act as a barrier to the absorption of topically applied chemicals. Therefore, the penetration depth of some drugs such as vaccines with a molecular weight greater than $500 \mathrm{Da}$ is limited by the SC [27]. An alternative to overcome such a problem is to create pores in the SC. In photothermal ablation of soft tissues, heating of chromophores mainly water content will rapidly vaporize the water within the tissue, hence causing a microexplosion in the form of phase explosion and the tissue is removed. The amount of ablated tissue depends on the fluence, heat deposited per unit volume and the laser pulse duration. As it will have discussed in the following sections, shorter the pulse duration, the smaller the tissue thermal damage controlled by the thermal relaxation time. An advantageous application of this point is the reduced thermal damage to surrounding areas of pores during drug delivery [4]. Also, it has recently been suggested that Dye-enhanced plasma-mediated ablation of the skin can be employed to increase the topical transdermal drug absorption [28]. It is, however, emphasized that photomechanical effect in its broad meaning may not necessarily be able to distinguish the differences between the different causes of such mechanical effect since it can be due to an acoustic, physical (e.g. bubble boundary), thermal, plasma and shock wave or indeed all but with different degree of participation at different time scale. Therefore, here the term photothermome- 
chanical effect is used to distinguish it from pure photothermoacoustic effect which is primarily applies in the thermoelastic domain. Indeed, the latter has been used for investigating photomechanical drug delivery using IR laser [29] [30] [31] [32].

Our previous studies showed the potential application of PLGA for carrying betamethasone [33], continuous $\mathrm{CO}_{2}$ laser delivery of PLGA/CS containing drug model [18], and laser thrombolysis with PLGA/CS containing tPA (Tissue Plasminogen Activator) drug [34]. Following these works, we report the dynamic study and visualization of cavitation bubbles containing PLGA/CS encapsulated fluorescein sodium nanoparticles ( NaFl NPs) utilized as drug model for delivery using a pulsed laser. Our early research has shown that photomechanical delivery (PD) is based on the generation of a cavitation or vapour bubble in a liquid medium due to high absorption of laser energy. Upon expansion and collapse of these bubbles the material (e.g. drug) are then displaced within the medium (i.e., biogelatin). During the first stage, the drug nanoparticles are transferred by cavitation bubbles and during the second stage when the bubbles collapse, the transfer is also enhanced by acoustic waves. However, at much higher irradiance level, LITB plays a dominant role.

\section{Materials and Methods}

\subsection{PLGA Containing NaFI NPs}

To fabricate these NPs, W/O/W double emulsion solvent evaporation surface coating method was employed as described [35]. For this, $3 \mathrm{ml}$ of deionized aqueous $\mathrm{Na} \mathrm{Fl}\left(\mathrm{C}_{20} \mathrm{H}_{10} \mathrm{Na}_{2} \mathrm{O}_{5}\right)$ (salt 4535-KGaA) with molecular weight of 376.28 $\mathrm{g} / \mathrm{mol}$ was used as drug model (Merck, Germany). This was then poured into 15 $\mathrm{ml}$ of dichloromethane (DCM) solution (Merck, Germany) containing $300 \mathrm{mg}$ of PLGA (50:50, Resomer RG $504 \mathrm{H}, \mathrm{Mw}$ 48000, Bohringer Ingelheim, Germany) and emulsified by a sonicator (tecna6-Tcno-GA - -S.P.A) to obtain a W/O emulsion. The emulsion was the added to $30 \mathrm{ml}$ of $1 \mathrm{wt} \%$ of polyvinyl alcohol (PVA, Mw 22000, Merck), and sonicated again to produce W/O/W emulsion. $300 \mathrm{ml}$ of $0.5 \mathrm{wt} \%$ of PVA was added to the emulsion and stirred mechanically. The solution was evaporated for $18 \mathrm{~h}$ and stirred at $250 \mathrm{rpm}$ to remove the solvent from the emulsified suspensions. The PLGA-NaFl NPs solution was centrifuged (Sigma, 3K30, RCF 25568, speed 16500 with rotor $12150 \mathrm{H}$, Germany) for 20 min to separate the NPs from the solution. The NPs were rinsed with distilled water followed by centrifuging number of times to remove the PVA and residual solvent. The NPs were then filtered through membrane filters to omit large sub-micron particles. Finally, the product dried at freeze dryer (Chaist, Alpha 1-2 LD plus, Germany) for further storage and use.

\subsection{CS Coated PLGA/NaFI NPs}

$300 \mathrm{mg}$ low molecular, $80 \%$ - 85\% deacetylation chitosan solution (Merck) was dissolved in $150 \mathrm{ml}$ of $1 \%$ acetic acid solution. $150 \mathrm{ml}$ of the $0.1 \mathrm{wt} \%$ CS solution 
and $150 \mathrm{ml}$ of $0.5 \% \mathrm{wt} \%$ PVA solution, instead of $300 \mathrm{mg}$ as above, were added to the prepared $\mathrm{W} / \mathrm{O} / \mathrm{W}$ emulsion. In order to enhance the CS coating on PLGA/NaFl NPs, the $\mathrm{pH}$ value of the emulsion was kept between 6 - 7 during the solvent evaporation.

\subsection{Biogelatin Model}

To model the tissue, $2 \mathrm{~g}$ of the commercial porcine skin biogelatin (3.5\% Gelatin-175 Bloom-Sigma Chemical, Type A) was mixed with $20 \mathrm{ml}$ of deionized water and then heated to $60^{\circ} \mathrm{C}$ with a magnetic stirrer. The final solution had a pale-yellow colour. For the experiment, the solution was poured into $30 \times 30 \times$ $50 \mathrm{~mm}$ vessel and after solidification a thin layer $(\approx 0.5 \mathrm{~mm})$ of CS@PLGA/NaFl NPs solution was applied as drug model covering the biogelatin surface.

\section{Characterization}

\subsection{Zeta Potential}

Laser light scattering (DynaPro ${ }^{\circledR}$ NanoStar) was used to determine the hydrodynamic size of NPs in aqueous solution at $25^{\circ} \mathrm{C}$ and zeta potential measurement (Zetasizer Zs, Malvern, UK) of NPs samples was made by suspending the freeze-dried $\mathrm{NPs}$ in $5 \mathrm{ml}$ deionized water.

\subsection{Microscopy}

\subsubsection{SEM}

Scanning electron microscopy (Vega 2, Tescan, Check) was used to determine the shape and surface morphology of the samples. Small amount of sample was placed on a double-sided tape attached on a metallic sample stand and coated by a thin layer of gold in vacuum chamber. The test as carried out three times and the results were presented as mean and standard deviation for three readings ( $\mathrm{n}$ $=3$ ). Significance in data between various process variables was assessed using all data points over multiple batches via student's $t$-test and one-way ANOVA with post-test. $\mathrm{P}$ value of $<0.5$ was considered significant.

\subsubsection{TEM}

Transmission electron microscopy (Philips CM 10, HT $100 \mathrm{k}$ ) was also used to study the morphology of NPs. The solution of NPs was placed on a carbon-coated 200 mesh size copper grid then $2 \mathrm{wt} \%$ of phosphatangstic acid was used to stain the NPs on the copper grid. The observation was done after the NPs were air-dried at room temperature.

\subsubsection{AFM}

The AFM with a Dualscope/Rasterscope system (C26, DME, Denmark) was utilized for imaging. The microscope was equipped with a scanner with maximum $\mathrm{XY}$ scan range of $50 \times 50 \mu \mathrm{m}$ and a $\mathrm{Z}$ range of $2.7 \mu \mathrm{m}$ and was operated by a scan Master (95-50E), a real-time closed-loop scanning control system which allows an accurate measurement, repositioning, and zooming in on selected features. 
The images were obtained using silicon nitride cantilevers with high-aspect ratio conical silicon tips with the force constants of $0.1 \mathrm{~N} / \mathrm{m}$ for contact mode imaging.

\subsubsection{FTIR Spectroscopy}

The FTIR spectroscopy (Nicolet, Magna IR Spec. 550, USA) of samples were performed using spectrum analyzer at $4 \mathrm{~cm}^{-1}$ resolution where $5 \mathrm{mg}$ of the NPs was mixed with $\mathrm{KBr}$ in order to identify the $\mathrm{CS}$ coating. The absorption spectra were recorded between $1000-4000 \mathrm{~cm}^{-1}$.

\section{Experimental}

In a simple experimental setup shown in Figure 2, a double-frequency pulse Nd:YAG laser (532 nm) (LG-LM1) with maximum output energy of $1.6 \mathrm{~J}$, pulse duration of $10 \mathrm{~ns}$ and pulse repetition frequency of $15 \mathrm{~Hz}$ was used for the experiment. The output beam with a $1 \mathrm{~mm}$ spot diameter was focused on the surface of biogelatin covered by a thin layer of drug model as described above. The time-resolved study was performed using a fast CCD camera (Panasonic Super Dynamic WV-CP450) connected to an optical microscope (Prior-UK), and a 2 $\mathrm{mW} \mathrm{He}-\mathrm{Ne}$ laser (Newport) which acted as a probe beam deflection. An ion

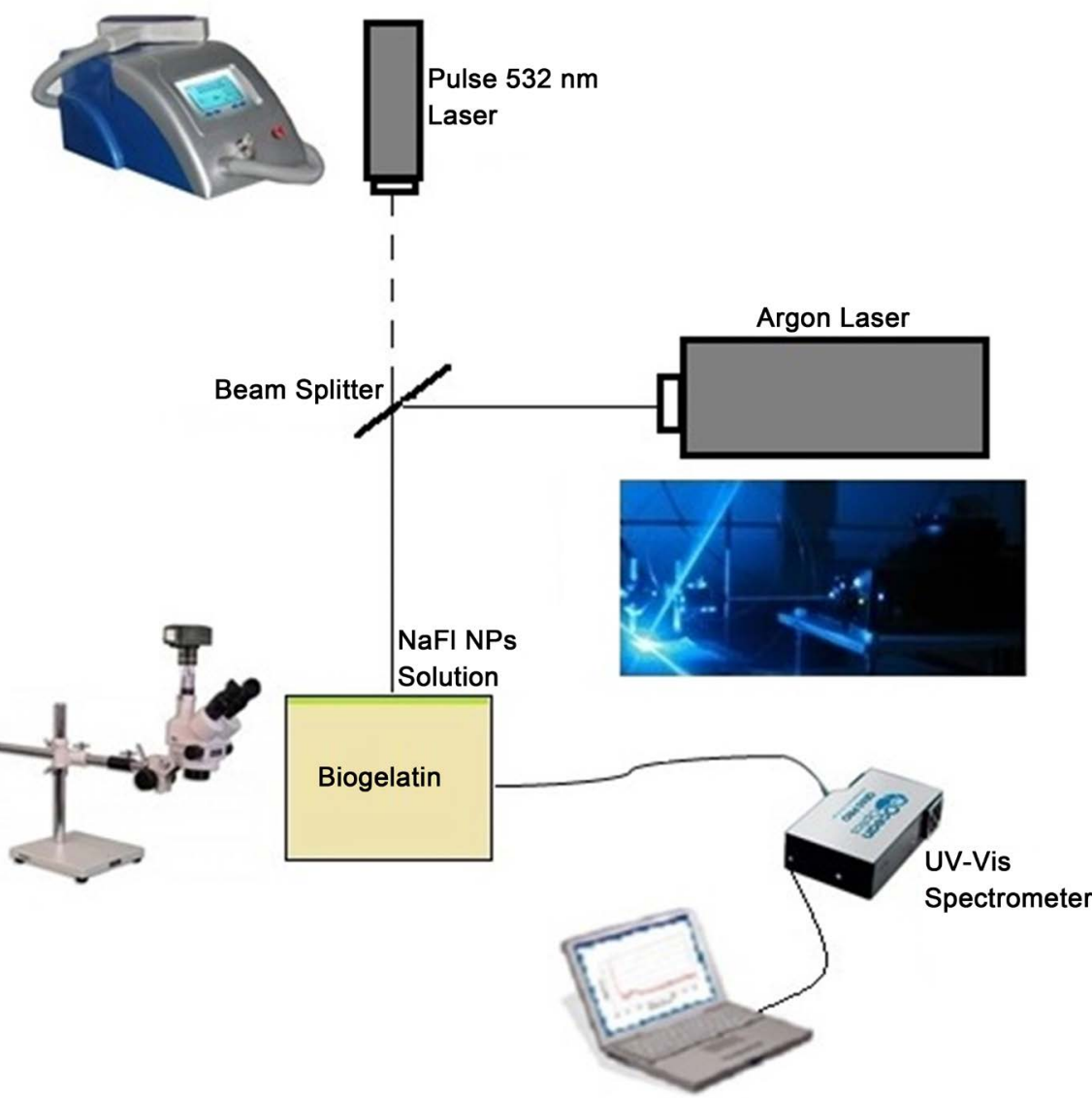

Figure 2. Experimental setup: pulse $532 \mathrm{~nm}$ is used to create cavitation in biogelatin and argon laser for fluorescence excitation after the laser irradiation. 
argon laser (Melles Griot-35MAP431) at $488 \mathrm{~nm}$ and $150 \mathrm{~mW}$ was used to induce fluorescence in samples after the irradiation (Figure 2). Visualization of the NaFl-loaded NPs driven by cavitation bubbles was done via the microscope. The fluorescence signals were collected by a $600 \mu \mathrm{m}$ core diameter optical fiber (LIBS-600-6-SR, Ocean Optics), which was connected to spectrometer (UV-Vis USB 4000, Ocean Optics) equipped with 2048-pixel linear silicon CCD array (14 $\mu \mathrm{m} \times 200 \mu \mathrm{m})$ with a detector range of $200-1100 \mathrm{~nm}$, optical resolution of 0.1 $10 \mathrm{~nm}$ FWHM for analysis and the output was then displayed on computer.

\section{Results}

Table 1 presents the results of synthesized NPs where the positive value of $9.6 \pm$ 0.008 for PLGA/CS indicates the formation of CS layer on the surface of PLGA NPs. This is also confirmed by the hydrodynamic diameters of $356 \pm 1.26 \mathrm{~nm}$ for $\mathrm{NaFl} / \mathrm{PLGA} / \mathrm{CS}$ compared to $248 \pm 0.77 \mathrm{~nm}$ of NaFl/PLGA NPs with the corresponding polydispersion index (PDI) values of 0.431 and 0.312 respectively, which shows the acceptable distribution. The increase of PLGA/CS hydrodynamic diameter with CS concentration can be due to increased viscosity of CS as well as other interactive forcers taking place at both ends i.e., attractive and van der Waal' force between the initial CS monolayer and PLGA surface and between the first CS monolayer and the subsequent layers due to for example hydrophobic interactions.

Figure 3 indicates the SEM of $\mathrm{NaFl}$ encapsulated by PLGA Figure 3(a) and $\mathrm{NaFl} / \mathrm{PLGA} / \mathrm{CS}$ NPs (Figure $3(\mathrm{~b})$ ) respectively with almost spherical geometrical shape. The spherical shape of the NPs is also illustrated by TEM micrographs. Figure 3(c) clearly, shows the shape and distribution of different sizes of PLGA NPs followed by encapsulation of drug model by PLGA in Figure 3(d) and finally, the drug encapsulated PLGA/CS NPs are shown in Figure 3(e).

The FTIR results of $\mathrm{NaFl}$ and $\mathrm{NaFl}$ encapsulated PLGA/CS NPs are shown in Figure 4 where the dominant peaks at 3084 and $1580 \mathrm{~cm}^{-1}$ correspond to $\mathrm{CH}$ and $\mathrm{C}=\mathrm{C}$ bonds present in the $\mathrm{NaFl}$ Figure 4(a), similarly, the peaks at 1091, 1759, and $3452 \mathrm{~cm}^{-1}$ in Figure 4(b) correspond to $\mathrm{CH}_{3}, \mathrm{C}=\mathrm{O}$ and stretching and vibrational modes of hydroxyl $(\mathrm{OH})$ bonds in PLGA respectively. The CS bonds are particularly shown at $1672,2854,2924 \mathrm{~cm}^{-1}$ corresponding to $\mathrm{C}-\mathrm{N}, \mathrm{CH}$ and $\mathrm{NH}_{3}$ bonds respectively. The 2D and 3D AFM pictures of drug loaded PLGA NPs are illustrated in Figure 4(c) \& Figure 4(d) and the corresponding drug loaded PLGA/CS NPs in Figure 4(e) \& Figure 4(f).

Differential Scanning Calorimeter (DSC) was used to address the quality of CS layer during the laser irradiation as a powerful thermal analyzer to measure the

Table 1. The polydispersion index, zeta potential and hydrodynamic size of the nanoparticles.

\begin{tabular}{cccc}
\hline PDI & Zeta Potential $(\mathrm{mV})$ & Hydrodynamic Diameter $(\mathrm{nm})$ & NPs \\
\hline 0.312 & -1.45 & $248 \pm 0.77$ & NaFl + PLGA \\
0.431 & 9.6 & $356 \pm 1.26$ & NaFl + PLGA + CS \\
\hline
\end{tabular}


(a)

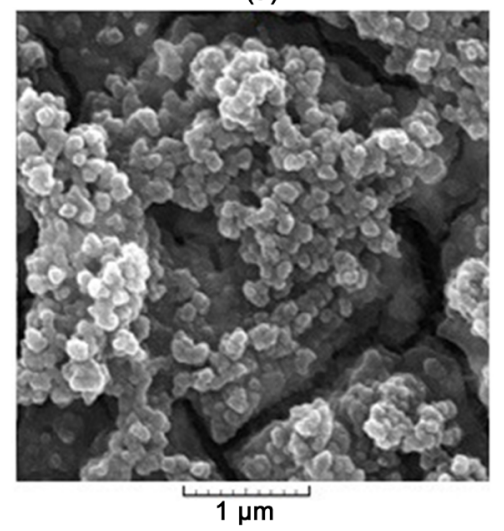

(c)

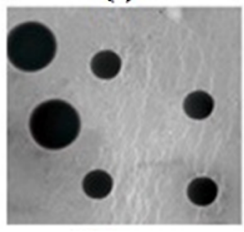

$150 \mathrm{~nm}$

PLGA NPs (d)
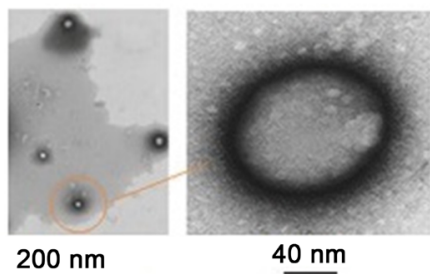

(b)

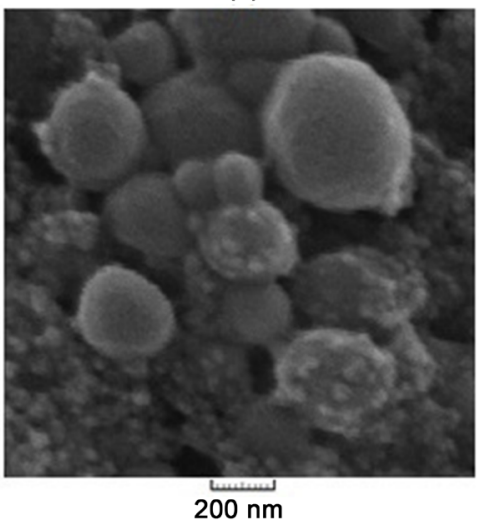

(e)

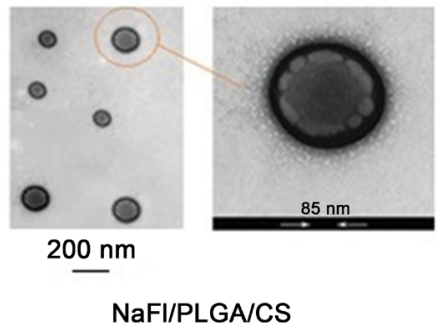

Figure 3. SEM of (a) NaFl encapsulated by PLGA and (b) NaFl/PLGA/CS NPs and TEM of (c) PLGA NPs, (d) NaFl/PLGA, and (e) NaFl/PLGA/CS nanoparticles.

(a): NaFI

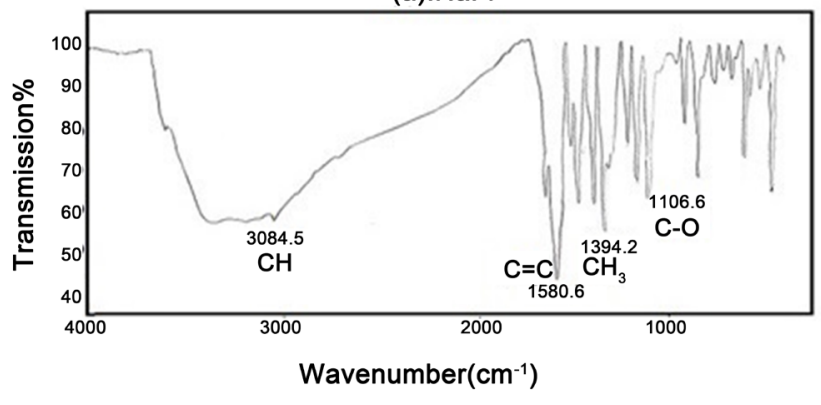

(b):NaFI/PLGA/CS

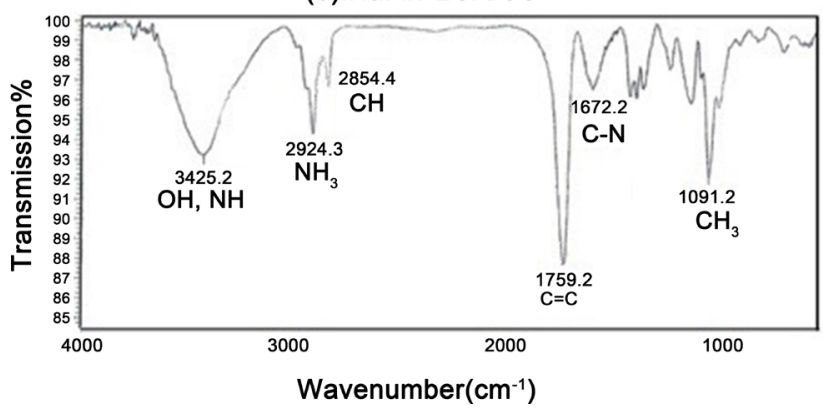

2D

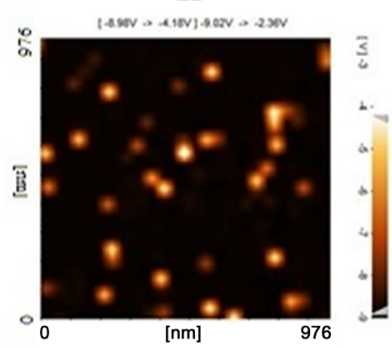

(c)

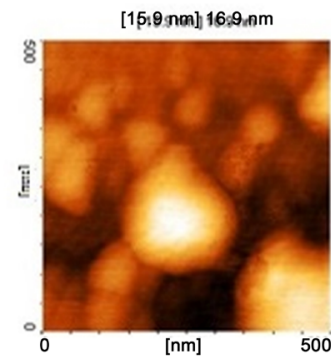

(e)

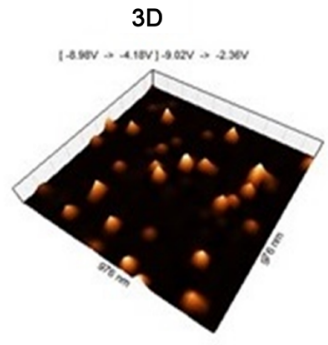

(d)
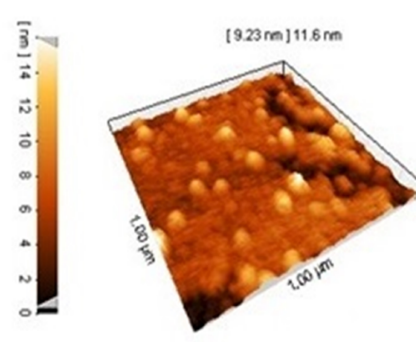

(f)

Figure 4. FTIR results of (a) NaFl and (b) NaFl encapsulated PLGA/CS NPs and AFM pictures of 2-D and 3-D drug loaded of (c, d) PLGA NPs and (e, f) PLGA/CS NPs.

energy absorbed or released as a function of time or a controlled temperature profile. The results in Figure 5 indicate the glass transition of $160^{\circ} \mathrm{C}$ for CS (a) and 
$55^{\circ} \mathrm{C}$ for PLGA in (b) respectively. The thermal degradation points of $270^{\circ} \mathrm{C}$ and $250^{\circ} \mathrm{C}$ are obtained for CS (a) and PLGA/CS (b) with their corresponding exothermic peaks at $300^{\circ} \mathrm{C}$ and $235^{\circ} \mathrm{C}$ respectively. This implies that the PLGA/CS NPs are relatively easier to thermal degradation and hence more susceptible for drug release compared to CS alone.

It is known that [36] that CS has mucoadhesive properties due to molecular attractive force formed by electrostatic interaction between positively charged CS and negatively charged surfaces. This is explained by number of factors mainly including: strong hydrogen bonding groups such as $-\mathrm{OH},-\mathrm{COOH}$, strong charges, high molecular weight, sufficient chain flexibility and surface energy properties. It is thought that the electrostatic attractive force is the main force during the early stages of monomolecular adsorption layer formation. This process continuous despite a positively charged surface due to hydrogen bond $(\mathrm{N}-\mathrm{H})$ and van der Waal's force acting on the surface. In the case of high CS concentration, after the direct formation of first layer on PLGA, the subsequent layer will not possibly be in direct contact with PLGA layer. Therefore, the added layer will increase the repulsion force due to increase of like charges between CS chains but interact and attracted via hydrophobic interactions, van der Waal's forces and hydrogen bonds [37]. The zeta potential of PLGA/CS NPs tends to increase with initial CS concentration until it reaches the plateau where it remains constant. It has been shown that the adsorption of CS on PLGA at high concentration $\left(0.4-0.6 \mathrm{gl}^{-1}\right)$ does not affect the zeta potential [36]. Prior to using drug model-loaded NPs, an experiment was carried out to test the interaction of laser with biogelatin as seen in Figure 6(a). The time-resolved dynamic study showed the formation of cavitation at $\approx 5 \mathrm{Jcm}^{-2}$ beneath the surface of biogelatin, which gradually expanded and finally the initial bubble detached after about 50 ms. A $2 \mathrm{~mW}$ He-Ne laser was used as a probe to visualize the depth of cavity as illustrated in Figure 6(b). The results of bubble formation as s function of pulse number at relatively low energy of $50 \mathrm{~mJ}$ corresponding to $6.5 \mathrm{Jcm}^{-2}, 640$ $\mathrm{MWcm}^{-2}$ and pulse frequency of $5 \mathrm{~Hz}$ are given in Figure 6(c). It demonstrates that the number of bubbles increases with increasing the pulse number and that the drilled channel widens accordingly. In practical term this certainly would

(a): CS

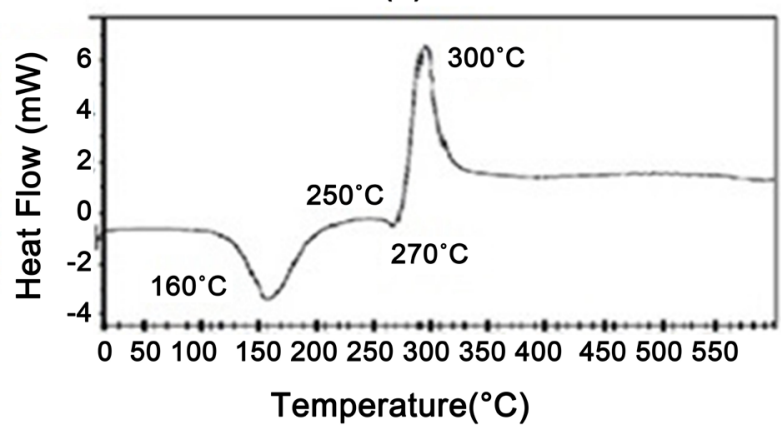

(b): PLGA + CS

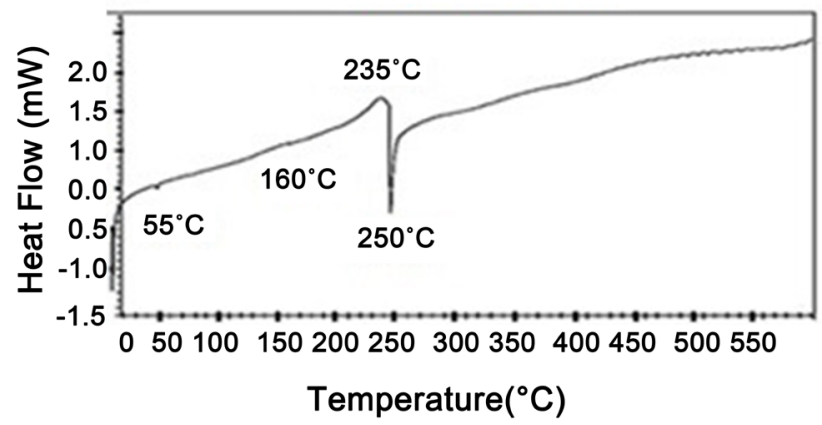

Figure 5. DSC results for glass transition of $160^{\circ} \mathrm{C}$ for CS (a) and $55^{\circ} \mathrm{C}$ for PLGA (b), and the thermal degradation points at $270^{\circ} \mathrm{C}$ for CS (a) and $250^{\circ} \mathrm{C}$ for PLGA/CS (b). Note that the exothermic peak for CS is reduced from $300^{\circ} \mathrm{C}$ to $275^{\circ} \mathrm{C}$ for PLGA/CS. 
(a)

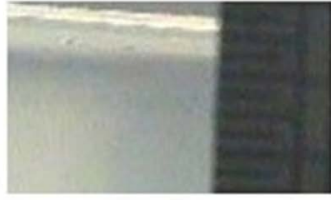

$\mathbf{t}=\mathbf{0 s}$

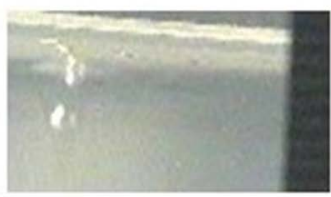

0.1

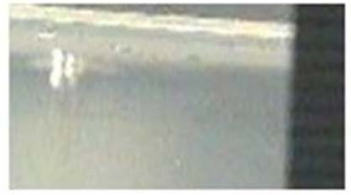

0.02

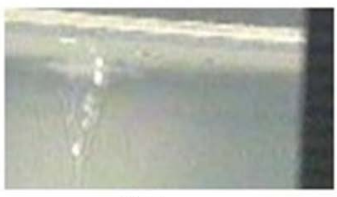

0.5

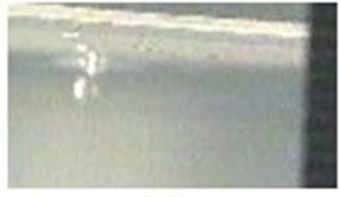

0.05

(b)
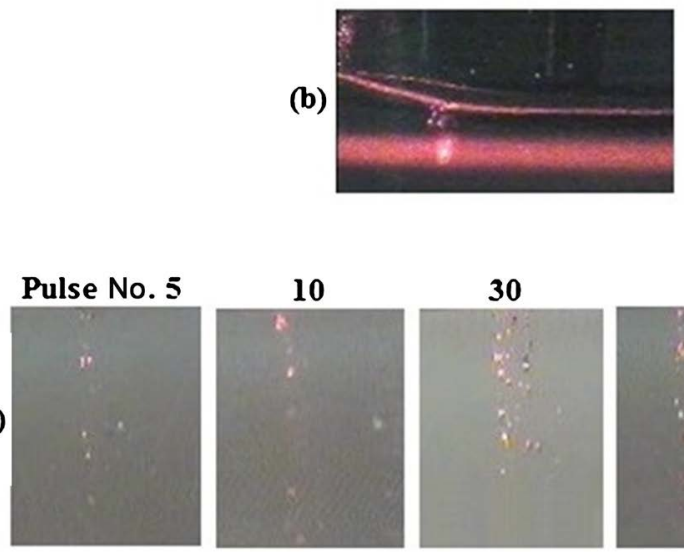

10

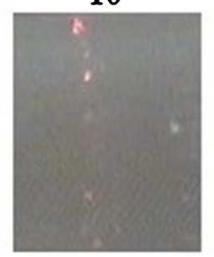

30

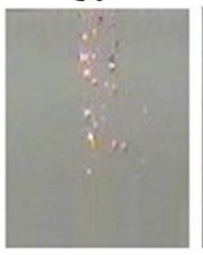

40
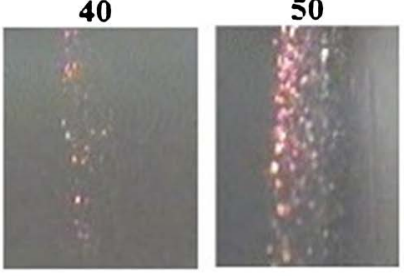

$6.5 \mathrm{Jcm}^{-2}$

Figure 6. (a) Time-resolved sequences of cavitation formation in biogelatin at $5 \mathrm{Jcm}^{-2}$; (b) Depth of cavitation is visualized by probe He-Ne laser; and (c) Bubble formation as a function of pulse number at $6.5 \mathrm{Jcm}^{-2}$. The bubble population increases by increasing number of pulses.

bear an implication on the amount of drug delivered within a tissue.

Figure 7 indicates an example of the irradiation of biogelatin at constant frequency of $2 \mathrm{~Hz}$ and at different low fluences of 3.5, 5, 6.5, 13 and $64 \mathrm{Jcm}^{-2}$ corresponding to $383-640 \mathrm{MWcm}^{-2}$ for F1 to F3 and 1.20 and $6.5 \mathrm{GWcm}^{-2}$ for F4 and F5 respectively. A blue filter was used as background to enhance the image contrast. The above values are in vaporization domain and below the laser-induced thermal breakdown (LITB) $\approx 1 \times 10^{10} \mathrm{Wcm}^{-2}$ i.e., thermionic emission threshold. As it is seen in Figure 7(a) by box (A), at lower input fluence of $\approx 3.5 \mathrm{Jcm}^{-2}$, the number of drug containing bubbles formed close to the biogel surface are comparatively less than those at higher values but gradually increase with increasing the fluence. It is also noted that the dimension of bubbles increases with fluence as shown by box (B). Figure 7(b) indicates that the bubbles formation beneath the surface were better observed when a pink filter was used. A magnified $(\times 10)$ portion of the gelatin shown in Figure 7 (c) illustrates the statistically varying bubbles dimension.

The experiment was repeated at higher fluences where LITB was observed as illustrated in Figure 8(a) where clearly the plasma is shown as white region and 


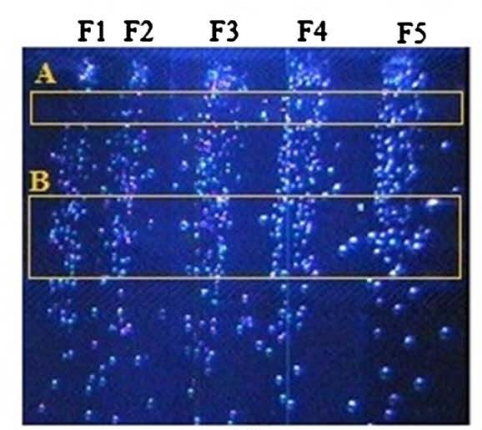

(a)

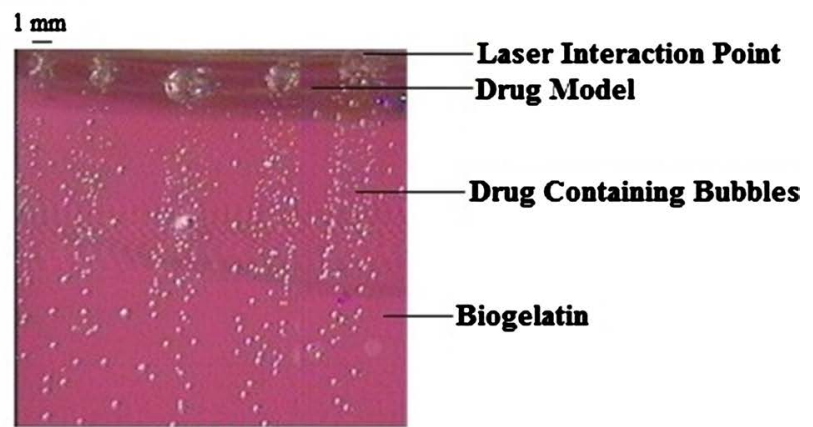

(b)

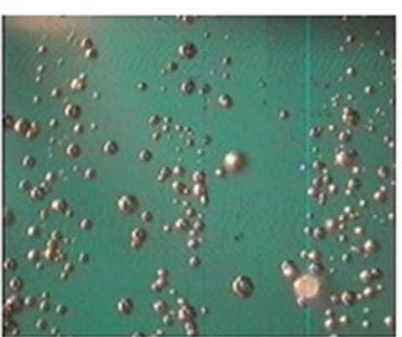

(c)

Figure 7. Cavitation bubbles produced in biogelatin at constant frequency of $2 \mathrm{~Hz}$ and at different fluences of 3.5, 5, 6.5, 13 and 64 $\mathrm{Jcm}^{-2}$ respectively. Box (A), demonstrates the number of drug containing bubbles formed close to the biogel surface are comparatively less than those at higher fluences. Box (B) shows that the dimension of bubbles increases with fluence.

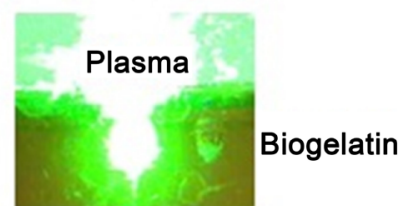

(a)

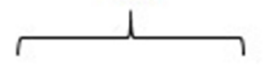
(b)
(c)
(d)
(e)
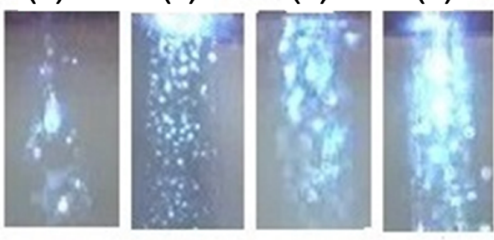

Argon laser-induced

fluorescence $(532 \mathrm{~nm}$ laser is

off)

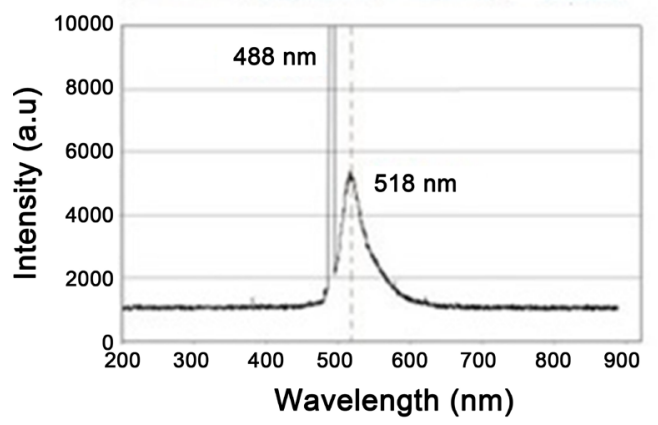

(f)

Figure 8. (a)Represents an example of LITB with plasma trapped within the cavity. The green colour is due to $532 \mathrm{~nm}$ wavelength, (b-e) Corresponding laser-induced fluorescence after irradiation at 1, 1.3, 1.9 and $2.5 \times 10^{10} \mathrm{Wcm}^{-2}$, and (f) An example of LIF emission of $\mathrm{NaFl} \mathrm{NPs}$ at green wavelength $(518 \mathrm{~nm})$ excited by $488 \mathrm{~nm}$ argon laser. 
the laser green light is entrapped inside the cavity. Once the experiment was finished, the sample was irradiated by argon laser to excite the NaFl NPs fluorescence to visualize the entrapped NPs track within the cavity. The Figures 8 (b)-(e) represents the irradiation at $1,1.3,1.9$ and $2.5 \times 10^{10} \mathrm{Wcm}^{-2}$ respectively and an example of the laser-induced fluorescence is given in Figure 8(f). Clearly, at higher irradiances, the regions of populated bubbles are significantly higher than the lower values due to more intense and energetic interaction. As seen, the brightness of argon laser strongly dominates the fluorescence signal and not distinguishable by naked eye but detected by spectrometer.

\section{Discussion and Analysis}

One of the challenges in the field of nanomedicine is accurate delivery of diagnostic and imaging agents to target. Since, normally drug concentration required for the therapy is toxic to healthy tissue, targeted or selective delivery of encapsulated drug is most desirable. Laser-induced drug delivery can be performed in variety of ways where a laser with high absorption coefficient and short pulse can be applied to impregnate the drug solution through the skin surface either with the epithelium removed or being intact. However, in some cases, it is possible to perforate both the drug solution and the soft tissue containing mainly water by an IR laser through which the drug is then transported and eventually diffused and absorbed by tissue network [14] [18] [38]. The above method can be enhanced when short pulse and low power laser is used to produce photoacoustic waves to push the remaining drug solution into the skin.

\subsection{Interaction Process}

Early research has shown the role of vaporization of agar gel [39]. Assuming the absorbed energy required by gelatin of infinitesimal thickness to reach the ablation threshold fluence $F_{t h}$ is,

$$
F_{t h}=\frac{(1-R) \Delta T \rho c}{\alpha}
$$

where for biogelatin $\alpha=1.43 \times 10^{4} \mathrm{~m}^{-1}$ at $532 \mathrm{~nm}$ is the absorption coefficient at given laser [40], wavelength, $R=\frac{\left(n_{m}-n_{a}\right)^{2}}{\left(n_{m}+n_{a}\right)^{2}} \approx 2 \%$ is the reflection of beam from the surface $n_{m}=1.33$ and $n_{a}=1$ are respectively the refractive indices of gelatin (assuming water) and air, $\rho=1200 \mathrm{~kg} \mathrm{~m}^{-3}$ is the density, $c=4180 \mathrm{~J}$ $\mathrm{kg}^{-1} \mathrm{~K}^{-1}$ is the specific heat capacity, $T_{c} \approx 100^{\circ} \mathrm{C}$ and $T_{o} \approx 20^{\circ} \mathrm{C}$ are the critical and initial temperatures respectively. Similarly, for PLGA/Ca containing drug model $\rho=300 \mathrm{~kg} \mathrm{~m}^{-3}, \mathrm{c}=2500 \mathrm{~J} \mathrm{~kg}^{-1} \mathrm{~K}^{-1}, \alpha \approx 700 \mathrm{~m}^{-1}$ the DSC results $\Delta \mathrm{T} \approx$ $230^{\circ} \mathrm{C}$. The energy balance shows that the evaporation of solution containing PLGA/CS NPs or the gelatin is achieved when the laser irradiance has reached the evaporation threshold, $I_{V}$ defined as

$$
I_{V}=\frac{\rho L_{v} \delta_{0}}{\tau_{p}}
$$


where $L_{V}=2260 \mathrm{~kJ} \mathrm{~kg}^{-1}$ is the latent heat of vaporization of water and $\delta_{0} \approx 70 \mu \mathrm{m}$ is the optical penetration depth. Table 2 and Table 3 indicate the calculated results for both PLGA/CS and biogelatin using the given corresponding data.

Since, in our experiment the laser fluences are above $\mathrm{F}_{\text {th }}$, a pure non-ablative thermoelastic stress wave does not apply, however, cavitation formation is possible. Consequently, the induced thermoacoustic pressure is mainly due to expansion and collapse of cavitation bubbles below $\mathrm{I}_{\mathrm{v}}$ but above the threshold vaporization, the recoil momentum of ablated material and shock waves are also added to the interaction process. In fact, there are number of non-linearities which should be considered at this stage 1) the coupling of temperature and pressure, 2) non-linearities in the propagation of a pressure pulse, and 3) optical saturation in the dye absorbing solution. Recently, it has been shown that Nd:YAG laser-induced photothermoacoustic effect can be used for photomechanical drug delivery where onset of significant hydrodynamic nonlinearity in the water for laser pulse irradiances in the $165 \mathrm{MWcm}^{-2}$ was observed [29]. The volume of the gelatin that is vaporized by the excess pulse energy above threshold can be estimated using

$$
V_{v}=\frac{A F_{t h}}{\rho L_{v}}\left[\frac{F}{F_{t h}}-1-\operatorname{In}\left(\frac{F}{F_{t h}}\right)\right]
$$

Figure 9 indicates the results obtained for the fluences used in Figure 7 and Figure 8 where a range of vaporized volume between $0.32 \times 10^{-3}-0.69 \mathrm{~cm}^{3}$ is obtained. The range of fluences are: zone (I) non-ablative and thermoelastic expansion only $\left(0-2.8 \mathrm{Jcm}^{-2}\right)$, zone (II) thermoelastic expansion cavitation and collapse $\left(2.8-18 \mathrm{Jcm}^{-2}\right)$, and zone (III) explosive vaporization including the cavitation bubbles and plume $\left(18-100 \mathrm{Jcm}^{-2}\right)$ where the heating is sufficiently high and the temperature is about critical temperature which causes a rapid homogeneous nucleation and expansion of vapour bubbles leading to explosive boiling carrying off the solid and liquid material fragments i.e., "Phase explosion" occurs, and zone (IV) LITB (100 - 255) $\mathrm{Jcm}^{-2}$ which includes number of

Table 2. The ablation threshold fluence with the corresponding values of energy, irradiance and the deposited heat on the PLGA/CS and biogelatin.

\begin{tabular}{ccccc}
\hline & $\begin{array}{c}\text { Ablation threshold } \\
\text { fluence } \mathrm{F}_{\mathrm{th}}\left(\mathrm{J} / \mathrm{cm}^{-2}\right)\end{array}$ & $\begin{array}{c}\text { Corresponding } \\
\text { energy }(\mathrm{mJ})\end{array}$ & $\begin{array}{c}\text { Corresponding irradiance } \\
\mathrm{I}_{\mathrm{th}}\left(\mathrm{GWcm}^{-2}\right)\end{array}$ & $\begin{array}{c}\text { Deposited heat } \\
\mathrm{Q}\left(\mathrm{GWcm}^{-3}\right)\end{array}$ \\
\hline PLGA/CS & 24 & 188 & 2.4 & 16.2 \\
Biogelatin & 2.8 & 22 & 0.28 & 0.34 \\
\hline
\end{tabular}

Table 3. The vaporization irradiance with corresponding energy and the threshold fluence applied on the PLGA/CS and biogelatin.

\begin{tabular}{cccc}
\hline & $\begin{array}{c}\text { Vaporizing irradiance } \\
\left(\mathrm{GWcm}^{-2}\right)\end{array}$ & $\begin{array}{c}\text { Corresponding } \\
\text { energy }(\mathrm{mJ})\end{array}$ & $\begin{array}{c}\text { Corresponding threshold } \\
\text { fluence } \mathrm{F}_{\mathrm{th}}\left(\mathrm{J} / \mathrm{cm}^{-2}\right)\end{array}$ \\
\hline PLGA/CS & 9.5 & 745 & 95 \\
Biogelatin & 1.8 & 141 & 18 \\
\hline
\end{tabular}




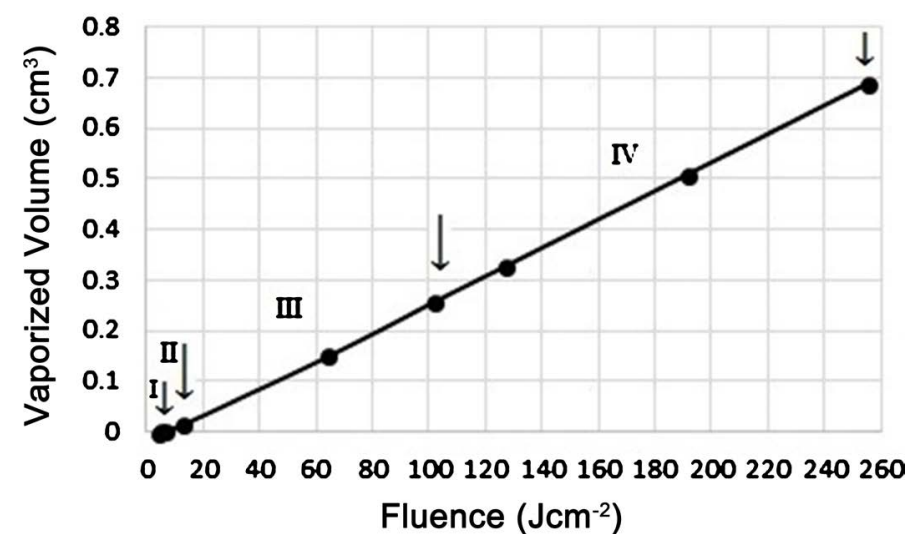

Figure 9. Vaporized volume of biogelatin at different fluences for various zones of interaction process: (I)-non-ablative thermoelastic, (II) thermoelastic with cavitation, (III) explosive vaporization with phase explosion, and (IV) LITB.

non-linearities introduced by high pressure and temperature. As an example, Figure 6(c) is well below vaporization, in Figure 7 F1, F2 and F3 are in zone II well below $I_{v}, F 4$ and F5 in zone III are above vaporization threshold and Figures 8(b)-(e) are within the LITB domain i.e., vaporization, plasma (see Figure 8(a)) and shock waves all present but at different time scales. Optical breakdown is an electronic and thermomechanical process which involves generation of plasma by either multiphoton (LIOB) or avalanche ionization (LITB) followed by explosive vaporization and mechanical expansion [41]. The origin of the thermoacoustic mechanical pressure lies in the fast deposition of energy in the focal volume resulting in dielectric breakdown which causes a rapid temperature and pressure rise within the plasma produced locally in the impurity centers. As a result, a fully developed compression wave of large amplitude i.e., shock wave is generated across which density, pressure and particle velocity change significantly [42] [43].

Figure 10(a) illustrates an example of bubbles formation at F5 $\left(64 \mathrm{Jcm}^{-2}\right)$ where it clearly indicates the front surface of bubble is opened and releasing the smaller bubbles (Figure 10(b)). A vapour-filled bubble is generated below the surface which expands with time. When the bubble expands, the shell thickness contracts and the concentration of gas near the bubble surface is increased and the rate of gas diffusion towards the bubble becomes greater than average. It is known that with transient cavities as in our case, great concentration of energy occurs towards the end of the contraction before the bubble breaks and producing large number of microbubbles. Indeed, in our early report [17], we showed that the bubble reaches a maximum size and then collapses; the maximum bubble size and the time scale for growth and collapse decrease as the fluence decreases. The bubbles become unstable in the final stage of collapse, dissipating through the generation of minute bubbles.

The maximum bubble radius can be determined using

$$
R_{\max } \approx\left(\frac{3 \omega^{2} F}{4 P_{0}}\right)^{1 / 3}
$$




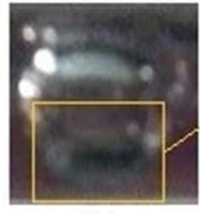

(b)

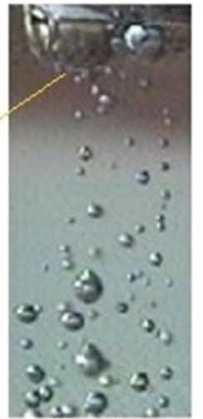

(a) (c)

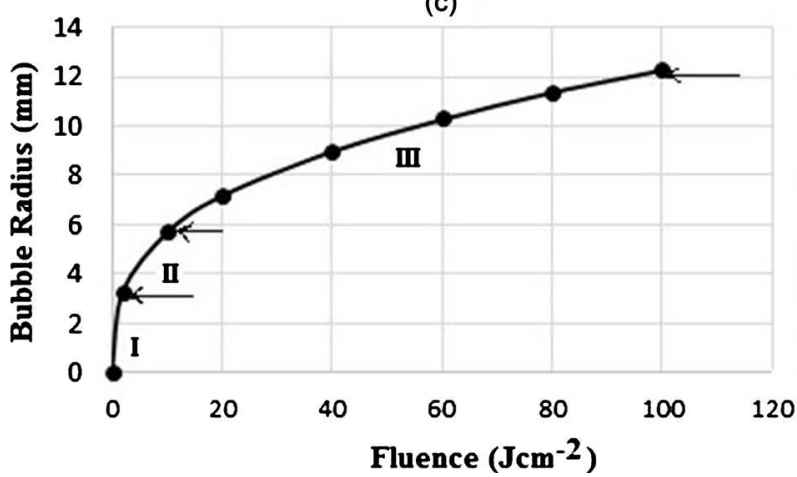

(d)

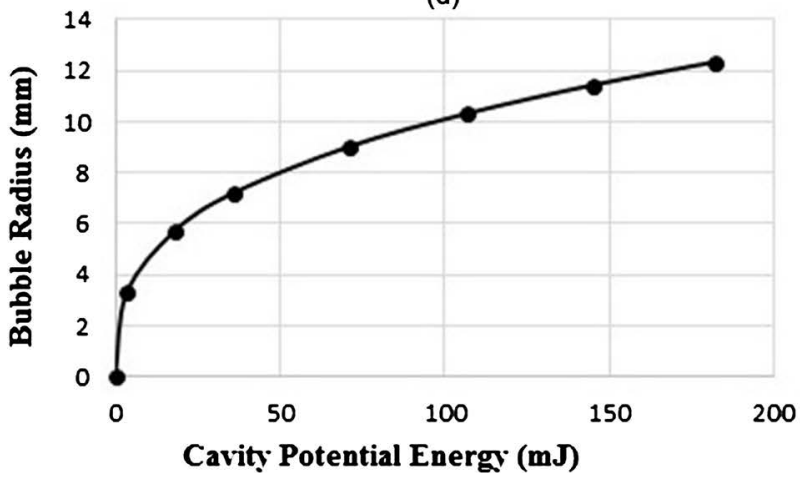

Figure 10. (a) An example of vapour-filled bubble formation at $65 \mathrm{Jcm}^{-2}$ beneath the biogelatin surface, and (b) Clos-up of (a) indicating the release of secondary microbubbles from distal end of the primary bubble. Variation of bubble radius with (c) Fluence and (e) Bubble potential energy. The turning point at about $3 \mathrm{Jcm}^{-2}$ is an indicative of onset of non-linearities.

where $\omega$ is the beam radius, $\mathrm{F}$ is the fluence and $P_{0}$ is the atmospheric pressure $\approx 10^{5} \mathrm{~Pa}$. It can be seen from Figure 10 (c) that $R_{\max }$ increases linearly with fluence in zone (I) i.e., thermoelastic but it gradually begins to deviate at the onset of cavitation in zone (II) and it continuous with the same trend for vaporization in zone (III). Despite the fact the behaviour of cavitation bubbles varies with the surrounding environment and materials and considering some experimental errors, the calculated values are in close agreement with the observation results. Clearly, as the fluence increases the more factors are involved during the interaction process and hence accounting for more complicated and non-linear behaviour of bubbles such as those in LITB in zone (IV). Thus, the Equation (4) is not used for this region. At its maximum radius the velocity of the bubble wall and hence of the liquid falls to zero and from the work done on the liquid the potential energy $E_{p}$ of the cavity is found to be:

$$
E_{p}=4 / 3 \pi R_{\max }^{3}\left(P_{0}-P_{v}\right)
$$

where $P_{s} \approx 2064 \mathrm{~Pa}$ is the saturated vapour pressure. The calculated results are shown in Figure 10(d) where a similar behaviour as Figure 10(c) is observed. The characteristic collapse (and growth) time for gas filled spherical bubbles is given by [44]

$$
\tau_{c}=0.91 R_{\max } \sqrt{\frac{\rho_{g}}{P_{0}-P_{v}}}
$$


This gives values in the range between $330 \mu \mathrm{s}$ and $1.35 \mathrm{~ms}$ for the corresponding $R_{\max }$ between 3 and $12 \mathrm{~mm}$ respectively.

At its maximum radius the velocity of the bubble wall and hence of the liquid falls to zero and from the work done.

\subsection{Thermal and Acoustic Confinement}

In the absence of vaporization as depicted in Figure $1(\mathrm{a}), \mathrm{F} \ll \mathrm{F}_{\mathrm{e}} \approx 18 \mathrm{Jcm}^{-2}$, the temperature increases due to transient heating produced by the energy deposition in the focal point volume leads to thermal expansion of the gelatin, as a result of which a momentum is set up within medium. Consequently, a rarefaction inside the heated volume is responsible for the compressive and tensile stresses. The thermal expansion directed into material towards the free surface generates negative stress. Temperature distribution in laser irradiated gelatin can be described by the Fourier heat conduction equation

$$
\rho c \frac{\partial T(r, t)}{\partial t}=K \nabla^{2} T(r, t)+Q(r, t)
$$

where $K=D_{t} \rho c \approx 5380\left(\mathrm{Wm}^{-1} \mathrm{~K}^{-1}\right)$ is thermal conductivity of gelatin and

$$
Q(r, z, t)=\alpha I(r, z, t)
$$

is the heat source indicating the rate of heat generated by the laser per unit volume $\left(\mathrm{Wcm}^{-3}\right)$. Considering an adiabatic case, $\mathrm{z} \gg 0$ and $\alpha \gg \beta$ where $\beta$ is the scattering coefficient, the solution to Equation (7) with a Guassian beam profile can be described as

$$
T(r, z)=\frac{\alpha I t}{\rho C} e^{-\alpha z} \cdot e-\frac{2 r^{2}}{\omega_{0}^{2}}
$$

The temperature increase will result in pressure distribution with a similar distribution as temperature, which acts as a source of pressure signal interacting with the medium. The thermal confinement is achieved if the pulse duration $\tau_{p}$ is smaller than the relaxation time $\tau_{r}$ where,

$$
\tau_{p}<\tau_{r}=\delta_{0}^{2} / 4 D_{t}
$$

where $\delta_{0} \approx \alpha^{-1} \approx 70 \mu \mathrm{m}$ is the optical penetration depth, $\mathrm{D}_{\mathrm{t}} \approx 1.4 \times 10^{-7} \mathrm{~m}^{2} \mathrm{~s}^{-1}$ [40] is the thermal diffusivity. Using these values it gives $\tau_{p}=10 \mathrm{~ns} \ll \tau_{\mathrm{r}} \approx 8.75$ $\times 10^{-3} \mathrm{~s}$. Also, for 1-D model, the thermal diffusion depth $\mathrm{X}_{\mathrm{T}}$ is determined by

$$
X_{T}=\sqrt{4 D_{t} \tau_{p}}
$$

Thus, $\delta_{0} \approx 70 \mu \mathrm{m} \gg \mathrm{X}_{\mathrm{T}} \approx 75 \mathrm{~nm}$. Therefore, in our case, the heat produced is confined and the adiabatic condition is satisfied i.e., the heat transport due to het conduction can be neglected. If the absorption coefficient of a liquid is so large that the optical penetration depth is much smaller than the radius of beam $\left(\delta_{0}\right.$ $\ll \phi / 2)$ then the shape of sound source can be considered as plane wave as in our case. Considering $\tau_{p} \ll$ acoustic transient time $\tau_{\mathrm{a}} \approx\left(\alpha \mathrm{c}_{\mathrm{a}}\right)^{-1} \approx 47 \mathrm{~ns}$ (i.e., the time required for the stress wave to propagate across the optical zone of energy 
deposition) where $c_{\mathrm{a}} \approx 1500 \mathrm{~ms}^{-1}$ is the speed of sound in water, and the acoustic penetration depth $\delta_{\mathrm{a}} \approx \mathrm{c}_{\mathrm{a}} \tau_{p} \approx 15 \mu \mathrm{m} \ll \delta_{0}$, then stress confinement is achieved. The photothermal ablative removal of material (Zone III and IV in Figure 9) from the gelatin surface can generate strong stress transients through the imparted recoil momentum of ablation plume as depicted in Figure 1. Although it is possible that only small mass of material is ablated, the short expulsion times and high ablation velocities can lead to large amplitude stress waves.

\subsection{Pressures}

The possible pressures during the interaction process are: 1) photothermoacoustic due to thermoelastic stress, 2) photothermal due to temperature distribution as described above, 3) photothermal ablative stress, and 4) shock wave. In our case, 1) does not apply because the applied fluences were above the threshold. The maximum ablative stress $\sigma_{\max } 3$ ) can be determined by

$$
\sigma_{\max }=V \rho \dot{x}
$$

where $\mathrm{V}$ is the ablation velocity and $\dot{\mathrm{x}}$ is the surface recession speed.

$$
\sigma_{\max }=V \rho I / \alpha \cdot \int_{0}^{t} I d t
$$

where $\mathrm{I}=\mathrm{F} / \tau_{p}$ is the laser irradiance. The peak stress is reached when $\int_{0}^{t} I d t=F_{t h}$, so

$$
\sigma_{\max }=\frac{V \rho F}{\alpha F_{t h} \tau_{p}}
$$

Thus, the maximum stress is directly related to laser fluence and $V=P / c_{a} \rho$. The product of stress and the rate of stress is a measure of mechanical energy propagating through a medium. Assuming for example pressure (in liquid) $\mathrm{P} \approx$ $10^{5} \mathrm{~Pa}, \rho=1200 \mathrm{kgm}^{-3}$, it gives $\mathrm{V} \approx 0.56 \mathrm{~ms}^{-1}$ in liquid. Using the Equation (14), values of 3.8, 30, 171 and $428 \mathrm{MPa}$ are achieved at ablation, vaporization, LITB thresholds and the final LITB point in Figure 9 corresponding to zones II, III and IV. As for the shock wave, it is assumed a spherical shape where it begins to form and propagates from a distance from the center of plasma equal to the length of the plasma. From the conservation of momentum

$$
P \approx P-P_{0} \approx U_{s} \rho_{s} U_{p}
$$

where $P$ and $P_{0}$ are peak and hydrodynamic pressure respectively, $U_{s}$ and $U_{p}$ are the shock and particle velocity respectively and $\rho_{0}$ is the density within the shock. Using the Rankine-Hugoniot expression to calculate the change in the internal energy behind the shock front [45]

$$
\Delta E=(1 / 2)\left(P+P_{0}\right)\left(V_{0}-V\right)
$$

$\Delta E$ is the change of internal energy between the shock and normal states and $V$ is the specific volume $1 / \rho$. Although, the energy deposited by the shock front in the gelatin may not be very high, the rate of deposition of energy is very sig- 
nificant. It is this shock energy which acts a source of photomechanical pressure. Thus, the total energy deposited is

$$
\Delta E_{T}=\int_{r_{0}}^{\infty} 4 \pi r^{2} \rho(r) \Delta E(r) d r
$$

It is seen that by increasing the fluence, the total deposited energy increases hence a stronger shock wave is produced. It is noteworthy that the fluorescence emission is known to be sensitive to the environment where different molecules may well coexist. Part of the fluorescence changes with temperature rise due to change in the microenvironment. Also, the pressure wave that follows the shock front may contribute to the increase of the internal energy. Finally, it is believed that the more general traditional term "photomechanical" is not sufficient to differentiate the origin of such mechanical effects in such applications as they are all initiated by photon absorption. Thus, the nature of such photomechanical effects can be decisive and important in terms of nanomaterial engineering and fabrication as they can be made case sensitive by selecting different materials for more specific application and accurate results.

\section{Conclusion}

PLGA/CS nanoparticles containing fluorescein sodium as drug model were successfully fabricated and characterized for investigating as drug delivery cargo using pulsed double frequency laser and LIFS technique. The results showed that the encapsulated cavitation bubbles are transported by different mechanisms at below and above vaporization threshold where photothermomechanical force is the main mechanism involved in the process. The secondary microbubbles generated during the collapse phase play a key role in deeper transportation. However, based on DSC results at higher irradiances level, plasma and shock formation can exceed the nanoparticles melting temperature and cause undesirable damage. The LIFS demonstrated the fluorescence emission within the cavitation bubbles after irradiation, which confirms the possibility of laser-induced transport of drug nanoparticles. In our case, despite detection of fluorescence, the brightness of argon laser was too strong and dominated the weak fluorescence background. This work can be completed in future by investigating the subject at thermoelastic region, fast time resolved-dynamic study, attenuated excitation beam and drug release evaluation.

\section{Acknowledgements}

MEK acknowledges the research funding provided by AUT faculty grant program.

\section{Conflicts of Interest}

The authors declare no conflicts of interest regarding the publication of this paper. 


\section{References}

[1] Langer, R. and Tirrel, D. (2004) Designing Materials for Biology and Medicine. Nature, 428, 487-792. https://doi.org/10.1038/nature02388

[2] Van Nostrum, C.F. (2004) Polymeric Micelles to Deliver Photosensitizers for Photodynamic Therapy. Advance Drug Delivery Review, 13, 9-16.

https://doi.org/10.1016/j.addr.2003.07.013

[3] Kent, K.M. and Graber, E. (2012) Laser Tattoo Removal: A Review. Dermatology Surgery, 38, 1-13. https://doi.org/10.1111/j.1524-4725.2011.02187.x

[4] Scheiblhofer, S., Thalhmer, J. and Weiss, R. (2013) Laser Microporation of the Skin: Prospects for Painless Application of Protective and Therapeutic Vaccines. Expert Opinion Drug Delivery, 10, 761-773. https://doi.org/10.1517/17425247.2013.773970

[5] Puvvada, N. (2011) Nanomedical Platform for Drug Delivery. Journal Nanomedicine Nanotechnology, 2, 1-5.

[6] Yiv, Sh. and Uckun F. (2012) Lipid Spheres as Attractive Nanoscale Drug Delivery Platforms for Cancer Therapy. Journal Nanomedicine Nanotechnology, 3, 1-6.

[7] Khosroshahi, M.E., Ghazanfari, L., Hassannejad, Z. and Lenhert, S. (2015) In-Vitro Application of Doxorubicin Loaded Magnetoplasmonic Thermosensitive Liposomes for Laser Hyperthermia and Chemotherapy of Breast Cancer. Journal. Nanomedicine Nanotechnology, 6, 1-9. https://doi.org/10.4172/2157-7439.1000298

[8] Khosroshahi, M.E., Hassannejad, Z. and Arshai, R. (2015) Nanoshell-Mediated Targeted Photothermal Therapy of HER2 Human Breast Cancer Cells Using Pulsed and Continuous Wave Lasers: An in Vitro Study. Laser Medical Science, 30, 1913-1922. https://doi.org/10.1007/s10103-015-1782-X

[9] Loo, S.C., Ooi, C. and Boey, Y. (2005) Influence of Electron-Beam Radiation on the Hydrolytic Degradation Behaviour of Poly(Lactide-co-Glycolide) (PLGA). Biomaterials, 26, 3809-3817. https://doi.org/10.1016/j.biomaterials.2004.10.014

[10] Xu, Y. and Du, Y. (2003) Effect of Molecular Structure of Chitosan on Protein Delivery Properties of Chitosan Nanoparticles. International Journal of Pharmaceutics, 250, 215-226. https://doi.org/10.1016/S0378-5173(02)00548-3

[11] Chung, T.W. and Lu, Y. (2002) Growth of Human Endothelial Cells on Photochemically Grafted Gly-Arg-Gly-Asp (GRGD) Chitosans. Biomaterials, 23, 4803-4309. https://doi.org/10.1016/S0142-9612(02)00231-4

[12] Riviere, J.E. and Heit, M. (1997) Electrically-Assisted Transdermal Drug Delivery. Pharmaceutical Research, 14, 687-697. https://doi.org/10.1023/A:1012129801406

[13] Tachibana, K. and Tachibana, S. (2001) The Use of Ultrasound for Drug Delivery. Echocardiography, 18, 323-328. https://doi.org/10.1046/j.1540-8175.2001.00323.x

[14] Zharov, V. and Latyshev, A. (1998) Laser Ultrasonic Transport of Drugs in Living Tissues. Annals New York Academic Science, 858, 66-73. https://doi.org/10.1111/j.1749-6632.1998.tb10141.x

[15] Kodama, T. and Tomita, Y. (2000) Cavitation Bubble Behavior and Bubble-Shock Wave Interaction Near a Gelatin Surface as a Study of In-Vivo Bubble Dynamics. Applied Physics B, 70, 139-149. https://doi.org/10.1007/s003400050022

[16] Ogura, M., Sato, S. and Terakawa, M. (2003) Delivery of Photosensitizer to Cells by the Stress Wave Induced by a Single Nanosecond Laser Pulse. Japanese Journal Applied Physics, 42, L977-L982. https://doi.org/10.1143/JJAP.42.L977

[17] Dyer, P.E., Khosroshahi, M.E. and Tuft, S. (1993) Studies of Laser-Induced Cavitation and Tissue Ablation in Saline Using a Fibre-Delivered Pulsed HF Laser. Journal Applied Physics B, 56, 84-93. https://doi.org/10.1007/BF00325245 
[18] Mahmoodi, M., Khosroshahi, M.E. and Atyabi, F. (2011) Dynamic Study of PLGA/CS Nanoparticles Delivery Containing Drug Model into Phantom Tissue Using $\mathrm{CO}_{2}$ Laser for Clinical Applications. Journal Biophotonics, 4, 403-414. https://doi.org/10.1002/jbio.201000121

[19] Shangguan, H., Casperson, L. and Shearin, A. (1995) Photoacoustic Drug Delivery: The Effect of Laser Parameters on the Spatial Distribution of Delivered Drug. SPIE, 2391, 394-402.

[20] Khosroshahi, M.E. and Asemani, M. (2017) Dynamics Study and Analysis of Laser-Induced Transport of Nanoferrofluid in Water Using Fluorescein Isothiocyanate (FITC) a Fluorescence Marker. Journal Modern Physics, 8, 2219-2244. https://doi.org/10.4236/jmp.2017.814137

[21] Steverding, B. ((1974) Ignition of Laser Detonation Waves. Journal Applied Physics, 45, 4507-3511. https://doi.org/10.1063/1.1663810

[22] Paltauf, G., Reichel, E. and Schmidt-Graz, H. (1992) Study of Different Ablation Models by Use of High-Speed Sampling Photography. SPIE, 1646, 343-352.

[23] Kim, D., Ye, M. and Grigoropoulos, C. (1998) Pulse Laser-Induced Ablation of Different Liquids and Acoustic Transients Generation. Applied Physics A, 67, 169-181. https://doi.org/10.1007/s003390050756

[24] Jacques, S., Gofstein, G. and Dingus, R. (1992) Laser-Flash Photography of Laser-Spallation in Liquid Media. SPIE, 1646, 284-294.

[25] Emmony, D. (1985) Interaction of IR Laser Radiation with Liquids. Infrared Physics, 25, 133-139. https://doi.org/10.1016/0020-0891(85)90067-3

[26] Noack, J. and Vogel, A. (1999) Laser-Induced Plasma Formation in Water at Nanosecond to Femtosecond Time Scales: Calculation of Thresholds, Absorption Coefficients, and Energy Density. IEEE Journal Quantum Electronics, 35, 1156-1167. https://doi.org/10.1109/3.777215

[27] Bos, J. and Meinardi, M. (2000) The 500 Dalton Rule for the Skin Penetration of Chemical Compounds and Drugs. Experimental Dermatology, 9, 165-169. https://doi.org/10.1034/j.1600-0625.2000.009003165.x

[28] Cook, H., Stone, J. and Yu, F. (2016) Femtosecond Pulsed Laser Ablation to Enhance Drug Delivery Across the Skin. Biophotonics, 9, 144-154. https://doi.org/10.1002/jbio.201500120

[29] Mandelis, A., Baddour, N., Cai, Y. and Walmsley, R. (2005) Laser-Induced Photothermoacoustic Pressure-Wave Pulses in a Polystyrene Well and Water System Used for Photomechanical Drug Delivery. Journal Optical Society A, 22, 1024-1036. https://doi.org/10.1364/JOSAB.22.001024

[30] Xia, J., Kim, Ch., Jonathan and Lovell, F. (2015) Opportunities for Photoacoustic-Guided Drug Delivery. Current Drug Targets, 16, 571-581. https://doi.org/10.2174/1389450116666150707100328

[31] Tang, S., Chen, J., Samant, P. and Xiang, L. (2016) Photoacoustic Image-Guided Drug Delivery in the Prostate. Proceedings of the SPIE, 9709, 97090L.

[32] Zhang, Y., Yu, J., Kahkoska, A. and Gu, Z. (2017) Photoacoustic Drug Delivery. Sensors, 17, 1400. https://doi.org/10.3390/s17061400

[33] Khosroshahi, M.E., Enayati, M., Sharifey, S. and Tavakoli, J. (2007) Evaluation of Drug Release from PLGA Nanoparticles Containing Betamethasone. Biophotonics SPIE, 6633, 66331E.

[34] Mahmoodi, M., Khosroshahi, M.E. and Atyabi, F. (2011) Early Experimental Results of Thrombolysis Using Controlled Release of Tissue Plasminogen Activator 
Encapsulated by PLGA/CS Nanoparticles Delivered by Pulse $532 \mathrm{~nm}$ Laser. Digest Journal Nanomaterial and Biostrtucture, 6, 889-905.

[35] Chung, T., Wang, S. and Tsai, W. (2008) Accelerating Thrombolysis with Chitosan-Coated Plasminogen Activators Encapsulated in Poly-(lactide-co-glycolide) (PLGA) Nanoparticles. Biomaterials, 29, 228-237. https://doi.org/10.1016/j.biomaterials.2007.09.027

[36] Guo, C. and Gemeinhart, R.A. (2008) Understanding the Adsorption Mechanism of Chitosan onto Poly(lactide-co-glycolide) Particles. European Journal Pharmaceutics and Biopharmaceutics, 70, 597-604. https://doi.org/10.1016/j.ejpb.2008.06.008

[37] Sinha, V., Singla, A., Wadhawan, S. and Kaushik, R. (2004) Chitosan Microspheres as a Potential Carrier for Drugs. International Journal Pharmaceutics, 274, 1-33. https://doi.org/10.1016/j.ijpharm.2003.12.026

[38] Frantz, M., Mathezioic, F. and Stoffel, M. (1988) Transport of Biologically Active Material in Laser Cutting. Lasers Surgery Medicine, 8, 562-566. https://doi.org/10.1002/lsm.1900080604

[39] Rasgar, S., van Germet, M., Welch, A. and Hayes, L. (1988) Laser Ablation of Discs of Agar Gel. Physics Medicine Biology, 33, 133-140. https://doi.org/10.1088/0031-9155/33/1/012

[40] Viator, J. and Prahl, S. (1999) Laser Thrombolysis Using Long Pulse Frequency-Doubled Nd:YAG Lasers. Lasers Surgery Medicine, 25, 379-388. https://doi.org/10.1002/(SICI)1096-9101(1999)25:5<379::AID-LSM3>3.0.CO;2-B

[41] Vogel, A. and Busch, S. (1996) Shock Wave Emission and Cavitation Bubble Generation by Picosecond and Nanosecond Optical Breakdown in Water. The Journal of the Acoustical Society of America, 100, 148-153. https://doi.org/10.1121/1.415878

[42] Doukas, A.G., McAuliffe, D. and Flotte, T. (1993) Biological Effects of Laser-Induced Shock Waves: Structural and Functional Cell Damage In-Vitro. Ultrasound in Medicine and Biology, 19, 137-146. https://doi.org/10.1016/0301-5629(93)90006-A

[43] Brujan, E. and Vogel, A. (2006) Stress Wave Emission and Cavitation Bubble Dynamics by Nanosecond Optical Breakdown in a Tissue Phantom. Journal Fluid Mechanics, 558, 281-230. https://doi.org/10.1017/S0022112006000115

[44] Young, F.R. (1989) Cavitation. McGraw-Hill, Oxford.

[45] Doukas, A. and Zweig, A. (1992) Characterization of Plasma-Induced Shock Waves. SPIE, 1646, 275-283. https://doi.org/10.1117/12.137469 